\title{
POLÍTICAS DE DESARROLLO Y ORDENAMIENTO TERRITORIAL: UN ANÁLISIS ACERCA DE LA VINCULACION DE AGENCIAS INTERNACIONALES, ORGANISMOS GUBERNAMENTALES Y NO GUBERNAMENTALES Y POBLACIÓN INDÍGENA
}

\author{
Malena Inés Castilla ${ }^{12}$ \\ ${ }^{1}$ Instituto de Ciencias Antropológicas, Facultad de \\ Filosofía y Letras, Universidad de Buenos Aires, Argentina \\ ${ }^{2}$ Consejo Nacional de Investigaciones \\ Científicas y Técnicas (CONICET), Buenos Aires, Argentina
}

\section{Introducción}

La trayectoria vinculada al análisis del desarrollo como problemática, se presenta relevante en las investigaciones científicas, en general, y de las ciencias sociales, en particular, desde hace varias décadas. A partir de la segunda guerra mundial, el desarrollo comenzó a ser entendido y ejecutado en tanto política pública y tuvo el propósito de modernizar a los países que no habían alcanzado el estatus económico de aquellos estados capitalistas (Harvey 2007). Ahora bien, las condiciones para que los países se modernizaran, implicaban generalmente, que los mismos aceptaran ciertas normas, instituciones y formas de gobierno determinados.

A mediados de la década de 1960, diversos organismos de crédito internacional comenzaron a involucrarse en proyectos de desarrollo que incluían a las poblaciones rurales de los países del denominado "tercer mundo". De este modo, se creó un discurso del desarrollo a partir del cual se elaboraron conceptos, teorías y objetos. Es decir, parafraseando a Escobar (1998), el desarrollo había creado un tercer mundo en tanto categoría monolítica, ahistórica y esencialista. Dichos organismos se orientaron entonces, a intentar solucionar situaciones de pobreza rural, despolitizando y negando las causas que la provocaron, invisibilizando su historia, sus relaciones de explotación y desestimando la dominación económica y política que sobre ellos ejercen los países 'desarrollados' (Escobar 1998). Nos basamos en autores como Hobart (1993), Grillo (1997), Ribeiro y Escobar (2006) y Gudynas (2009) 
para explicar que los proyectos de desarrollo, en muchas ocasiones, ejecutan y ponen en juego promesas de soluciones que no solo son inalcanzables, sino que profundizan los problemas preexistentes.

En la Argentina, esta problemática no fue tratada por tales organismos hasta la década de 1980, dado que la preponderancia económica del país provenía de regiones como la pampeana, que garantizaban que este país se instalara a nivel mundial como proveedor de los productos agroganaderos. Luego del periodo dictatorial (1976-1983) que se estableció en el país al mando de las Fuerzas Armadas, con el retorno de la democracia en el año 1983, en el Noroeste argentino se implementaron proyectos para favorecer a los productores locales a partir de la cooperación de organismos públicos y organizaciones no gubernamentales; como el Instituto Interamericano de Cooperación para la Agricultura, el Instituto Nacional de Tecnología Agropecuaria, Instituto Nacional de Cultura Popular (INCUPO) Fundación por la Paz y la Justicia (Fundapaz), entre otros (Lattuada 2014).

Ahora bien, durante la década de 1990 se produjo a nivel mundial la implementación de una nueva corriente económica, a partir del denominado Consenso de Washington que promovía un sistema mundial capitalista con Estados retraídos frente a una economía neoliberal (Harvey 2004). Durante este período, en la Argentina se instalaron definitivamente los programas y políticas de desarrollo impulsados por agencias de crédito. Asimismo, se produjeron en la Argentina, durante la década de 1990, políticas privatizadoras, siendo esta una de las líneas de acción más importantes del modelo neoliberal impulsado por el Consenso. De este modo, la expansión de la frontera agrícola-ganadera se manifestó a partir de la venta de grandes extensiones de tierras para la inversión capitalista de empresas nacionales e internacionales.

En este escenario, las políticas de desarrollo comenzaron a planificarse desde un lugar competitivo y productivista, siendo los territorios el centro de la actividad capital. Cabe destacar que partimos de la noción de desarrollo entendiendo a la misma en relación con la desigualdad y el uso de la tierra. En este sentido, el desarrollo se presenta como un proceso conflictivo, que implica relaciones de poder desiguales garantizadas a partir de la institucionalización de políticas gubernamentales (Lapes de Souza 1995). El territorio, como analizaremos en el caso aquí propuesto, es el ámbito en el cual dichas relaciones de dominación se desenvuelven poniendo como protagonistas a las poblaciones de pequeños productores e indígenas como centro de tales políticas de intervención y apropiación (Manzanal et al. 2011). No podemos pensar al territorio y a sus actores, sin entenderlo como un bien en disputa, en el cual se desarrollan y determinan relaciones de poder y de clase (Schmidt 2014). En América Latina, en general, y en la Argentina, 
en particular, las políticas que fomentaron la expansión de la frontera agrícola-ganadera, así como los planes de desarrollo terminaron por desestructurar aquellos procesos de producción socioeconómicos vinculados a lo domestico y lo comunal (Baines 2004; Trinchero 2007).

El arribo de grandes capitales de la agroindustria a la Argentina se ha dado a partir de la expansión de las fronteras en zonas como el Noreste Argentino, sumado a las políticas activas que implementaron el Estado Nacional y los gobiernos provinciales y locales. Este movimiento ha generado actividades orientadas a la extracción de recursos naturales tales como madera, algodón, soja, entre otros, así como la explotación de mano de obra. Como resultado de ello, en la actualidad se presenta un escenario de acentuada desigualdad social y territorial en zonas como la provincia del Chaco [Ver imagen 1]. Esta situación, refleja el conflicto, las disputas y las alianzas entre los sectores sociales, económicos y políticos intervinientes en las negociaciones y resistencias que se producen frente al acceso a los recursos financieros y naturales. El territorio entonces se convirtió en el centro y en el ámbito en el cual se desarrollaron dichas disputas.

Imagen 1: Mapa de la Provincia del Chaco elaborado por el Instituto de geografía Nacional.

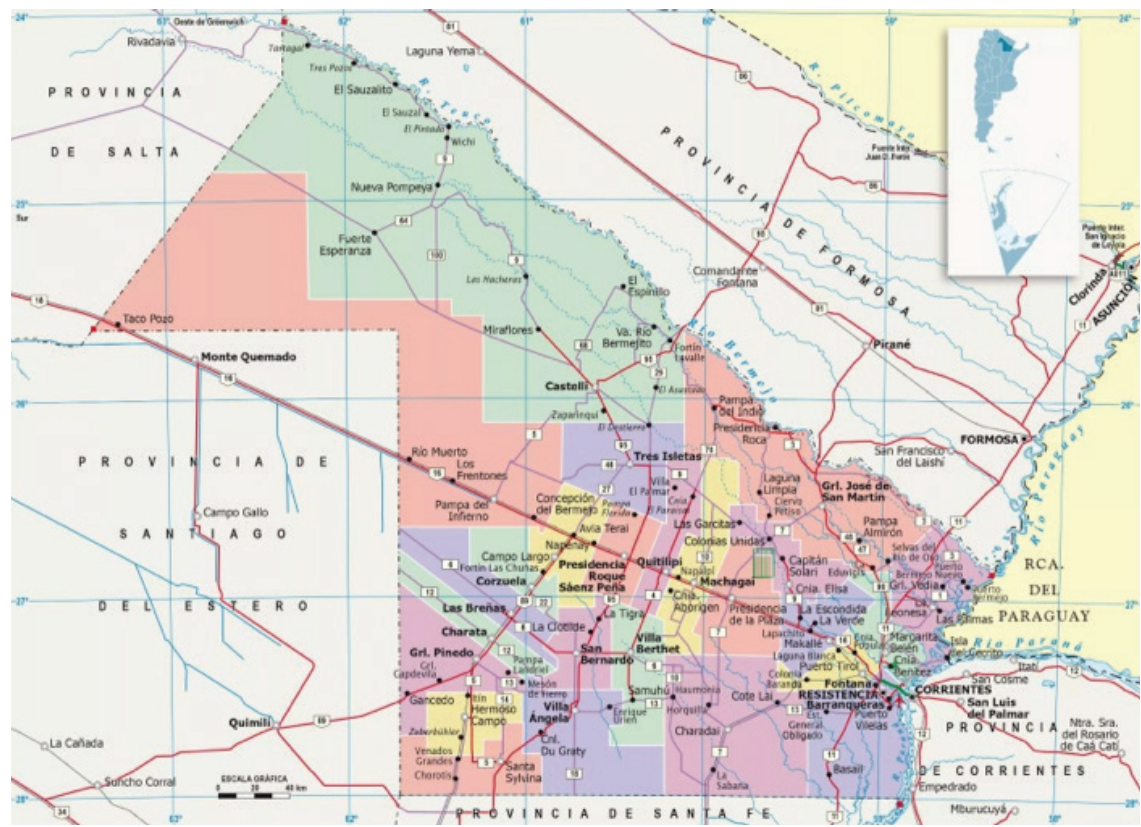

Disponible en: https://epicentrogeografico.com/chaco-2015-politico/ (Fecha de ultima consulta: 30 de octubre 2019)

Por tanto, en este artículo, nuestro objetivo es el de analizar aquellas vinculaciones que existen entre el empresariado transnacional y el Estado nacional, así como los diferentes niveles de gobierno y las fundaciones 
POLÍTICAS DE DESARROLLO Y ORDENAMIENTO TERRITORIAL:

UN ANÁLISIS ACERCA DE LA VINCULACION DE AGENCIAS INTERNACIONALES

ORGANISMOS GUBERNAMENTALES Y NO GUBERNAMENTALES Y POBLACIÓN INDÍGENA

y asociaciones civiles que se despliegan en el territorio e implementan diferentes estrategias que repercuten, intencionalmente o no, entre las poblaciones locales. Antes de continuar, nos parece importante expresar que este artículo es producto del trabajo realizado durante el proceso de investigación doctoral. La misma empezó en el año 2014 y se finalizó en 2019. Para ello, las técnicas desplegadas desde enfoques cualitativos y cuantitativos de carácter etnográficos fueron centrales para analizar los procesos a escala local y nacional, pero también las relaciones entre los diferentes sectores involucrados. Por tanto, gran parte de las entrevistas que están citadas en este documento son el resultado de encuentros en el trabajo de campo con múltiples actores. Tales entrevistas, son presentadas con nombres ficticios para preservar el anonimato de dichos sujetos.

\section{Políticas gubernamentales e inversión pública}

Tal como mencionamos anteriormente, a partir de la década de 1990, se consolidó en la Argentina el modelo económico y político de corte neoliberal. Este impulsó y garantizó la participación e inversión de capitales extranjeros a partir de la privatización no solo de bienes, servicios y empresas estatales, sino también de territorios fiscales. Durante este periodo, en la provincia del Chaco, se instrumentaron a partir de organismos gubernamentales del ámbito provincial, diferentes medidas y estrategias que garantizaron la instalación de empresarios del agro. Entre los años 1994 y 2007, la provincia perdió el 80\% de las tierras fiscales a manos de sociedades anónimas y empresarios dedicados a la producción agrícola y ganadera. Según los datos oficiales del Instituto de Colonización (IC), el Estado chaqueño pasó de tener 3 millones y medio de hectáreas en el año 1994 a 650 mil en diciembre del 2007 (Muñoz y Gallo 2012). Se generó en esta década la expansión de la soja genéticamente modificada, lo cual no solo significó la reconversión productiva, sino que forzó, una vez más, la expansión de la frontera agrícola. Es decir, la política estatal estuvo asociada a la desregulación económica y brindó la posibilidad de que se implante un mercado internacional y nacional en zonas rurales. Cabe destacar que el IC, se creó tras implementarse la Ley N. ${ }^{\circ} 2913$ en 1984, a partir del Régimen de Tierras Fiscales decretada por el Poder Ejecutivo de la provincia chaqueña. Dicho organismo es el encargado de manejar el patrimonio territorial de todos los ciudadanos. Es decir, el IC administra y regula las tierras que no poseen dueños privados y son propiedad del Estado. En tal sentido, los dos primeros artículos de la Ley sancionada por la Cámara de Diputados de la provincia del Chaco establecen: 
"Artículo 1.- Se regirán por las disposiciones de la presente Ley, las tierras rurales provinciales y las privadas que por cualquier título se incorporen al dominio del Estado provincial, con la excepción de las tierras afectadas a destinos y reservas específicas.

Artículo 2.- las tierras fiscales a que se refiere la presente Ley serán incorporadas al proceso productivo, propendiendo a su privatización por la adjudicación en arrendamiento con opción a compra o por venta" (Ley N. 2913 "Régimen De Tierras Fiscales" de la Constitución de la provincia del Chaco).

Entendemos que los objetivos de dicho organismo tienen que ver con la privatización de las tierras, para incorporarlas al proceso productivo -tal como señala en el artículo 2-. Es nuestra intención, partir de esta legislación para dar cuenta de los procesos a través de los cuales dicho ente se desenvuelve en la localidad que aquí investigamos. Por ello, pretendemos exponer los relacionamientos que se producen, entre los habitantes indígenas y otros actores de la zona, a raíz de las diferentes políticas públicas que generan nuevas modalidades a partir de las cuales se habita y utiliza el territorio. En este sentido, dichas políticas fueron implementadas en los territorios de la provincia, dando lugar a diversas denuncias por corrupción y por la venta indiscriminada de hectáreas que, en muchos casos, poseían propietarios. Al respecto, El Centro de Estudios e Investigación Nelson Mandela ${ }^{1}$ elaboró diversos informes en los cuales daban cuenta de las irregularidades que existían en el IC. Las denuncias fueron realizadas por organizaciones de pequeños productores y de indígenas de la zona que resultaron involucradas en tales anomalías. Entre los hechos denunciados se encontraban inconsistencias tales como: la adjudicación de tierras a personas fallecidas, otorgamiento de grandes extensiones a aquellos que no poseían animales o cultivos, así como las denuncias que detallaban que los funcionarios y técnicos del Instituto administraban -de modo arbitrario- hectáreas habitadas y generaban desalojos de familias de estas tierras. Además del informe presentado por este centro de estudios, un documento elaborado por el Frente Nacional Campesino (FNC), entregado en diciembre de 2016 al presidente del IC, establecía lo siguiente:

“En las adjudicaciones y en los trabajos de mensuras del Instituto de Colonización se realizaron varios casos de despojos de potreros, cercos, aguadas, corrales, chiqueros y viviendas de campesinos por parte de funcionarios[...] presiones y extorsiones contra campesinos de parte de determinados funcionarios políticos y del Instituto de Colonización, para que firmen conformidad de adjudicaciones irregulares y familias que quedarían sin tierra porque no figuran en los listados de adjudicaciones del Instituto de Colonización" (Diario Norte 2016). 
POLÍTICAS DE DESARROLLO Y ORDENAMIENTO TERRITORIAL:

UN ANÁLISIS ACERCA DE LA VINCULACION DE AGENCIAS INTERNACIONALES

ORGANISMOS GUBERNAMENTALES Y NO GUBERNAMENTALES Y POBLACIÓN INDÍGENA

Ahora bien, entre las denuncias encontramos que dicho organismo parecería favorecer a grandes empresarios que se dedican a actividades agropecuarias. Tal beneficio lo obtienen en detrimento de la calidad de vida de los habitantes indígenas que ocupan esos territorios, según afirman los denunciantes. Ricardo, un pequeño productor de Pampa del Indio nos contaba lo que sucede en la zona donde vive:

“El Instituto de Colonización lo que hace es que viene y verifica que vivís acá, la vecina reconoce que vivís. Yo instituto tengo que dar visto bueno, tengo que certificar que no hay nadie, y veo que el campo está vacío y la casa abandonada... y yo justo me fui a buscar agua, y no me viste vos y crees que no estoy. Si, está abandonada, así de simple. Hay irregularidades y nosotros tenemos que hacer algo" (Ricardo, productor de Pampa del Indio, junio de 2012).

En el relato de este poblador, observamos cómo, a la hora de verificar si un terreno está habitado, el IC perjudica a los pequeños productores e indígenas de la zona. Las palabras del productor aluden a un hecho ocurrido a una familia que vivía en un lote lindante a los campos de un empresario. Debido a que dicha familia no quería vender a bajo costo su terreno al empresario, el IC obró a favor de este, y desalojó a la familia bajo la acusación de que no residían en esa propiedad. Es decir, el IC, usualmente, operativiza y legítima la expropiación de tierras y modifica los límites entre las tierras fiscales y las tierras privadas, los cuales convierten -selectivamente- a los territorios fiscales en privados.

Lo mismo ocurre con el Instituto del Aborigen Chaqueño (IDACH), creado en 1987 y oficializado por la Ley Aborigen N. ${ }^{\circ} 3258$, que posee un presupuesto destinado a la población indígena, siendo esta la que a través de elecciones selecciona un dirigente para la presidencia del instituto. Las políticas que este organismo ejecuta están destinadas, entre otras cosas, a realizar alambrados perimetrales, como solución a una supuesta problemática local. Estos alambrados tienen como finalidad delimitar espacialmente los territorios, para subdividir y establecer los límites geográficos de grandes extensiones de tierra. Además, se utilizan para encerrar ganado, lo cual favorece la productividad agrícola ganadera, según nos explicaba un empleado del instituto. Otra de las políticas llevadas a cabo por el IDACH, tiene que ver con la construcción y refacción de viviendas rurales. Generalmente, dichas viviendas se emplazan en los montes chaqueños, donde habitan la población indígena pertenecientes a diferentes pueblos ${ }^{2}$. Las casas construidas y refaccionadas por este organismo, son de material (ladrillos, cemento, etc.).

Ambas políticas implementadas por el Instituto (alambrados y viviendas) deberían beneficiar a la población a la que está destinado dicho organismo, 
es decir, a las comunidades indígenas de la provincia. Asimismo, tal como nos informaron funcionarios de esta dependencia gubernamental, la ejecución de tales políticas se debe realizar de acuerdo con un protocolo que establece la designación de la zona donde se aplicarán, para evitar situaciones de corrupción o arbitrariedades en su implementación.

Respecto a las políticas de alambrado perimetral, llevadas a cabo por el IDACH, consideramos -en relación con las denuncias y relatos de nuestros entrevistados- que no responden a las necesidades de las comunidades, sino que actúan en función de delimitar y geo-referenciar las tierras indígenas de las de los empresarios privados. Desde el IDACH, argumentan que el pedido de los alambrados es realizado por las comunidades, y que a partir del mismo se entregan los materiales que los propios peticionantes instalan. Como mencionamos anteriormente, según estas argumentaciones, la función de los alambrados es lograr que el ganado que poseen los indígenas no invada el terreno lindante. Según nuestros registros de campo y los entrevistados, en realidad son pocos los integrantes del pueblo qom de Pampa del Indio los que tienen animales en sus terrenos (por falta de agua y pasturas). Por tanto, dicha política buscaría, en realidad, beneficiar a los empresarios que utilizan los alambrados para delimitar sus hectáreas y diferenciarlas de las que habitan la población indígena. Según la percepción de Héctor, integrante qom de la localidad: "[...] acá hay más alambrados que indios" (entrevista realizada en agosto 2017).

Por tal motivo, este Estado promotor que garantiza facilidades a los inversionistas, permite el acceso y permanencia a los empresarios a partir de leyes, políticas y organismos destinados a estimular, facilitar y subsidiar a las actividades relacionadas (Manzanal, et. al 2010). Dicha promoción, además es garantizada, aún en la actualidad, a partir de la inversión de fondos públicos internacionales (tomados en deuda), como nacionales, para la ejecución de obras de infraestructura, fundamentalmente, y que son las que nos interesan resaltar en este artículo. Nos referimos a obras de infraestructura como las mejoras y pavimentaciones de rutas, implantación de sistemas de riego, instalación de acueductos, etc.

Ahora bien, dicho proceso trajo aparejado diversas consecuencias a nivel local para su población. En primer lugar, es importante mencionar que la privatización de las tierras, en muchas ocasiones se realizó sobre territorios ancestrales de la población indígena. En este sentido, se generó una política de expulsión que dio lugar a la migración de gran parte de la comunidad local indígena y campesina. Según los censos poblacionales, en la provincia del Chaco, entre 1991 y 2001, la población rural descendió en más de 60.000 habitantes (INDEC 1991; 2001). Asimismo, la población 
POLÍTICAS DE DESARROLLO Y ORDENAMIENTO TERRITORIAL:

UN ANÁLISIS ACERCA DE LA VINCULACION DE AGENCIAS INTERNACIONALES

ORGANISMOS GUBERNAMENTALES Y NO GUBERNAMENTALES Y POBLACIÓN INDÍGENA

que consiguió permanecer en sus tierras y hogares ha sido víctima de las transformaciones socioeconómicas, producto de la privatización de los montes, y los cambios en el hábitat natural por las fumigaciones y forestaciones. El boom productivo de los últimos años se expandió hacia el monocultivo de la soja transgénica, en primer lugar, y vacuna, en segundo lugar. Cabe destacar que, la producción de la soja permite, además, la generación de biocombustible para la exportación a países de Europa, fundamentalmente. Es en este contexto de precarización laboral y explotación de las tierras, que se implementaron en la provincia diferentes programas de desarrollo durante la década del 2000 (Baines 2001; Lattuada 2014). El objetivo en dichos programas era el de trabajar en aquellos sectores donde la pobreza afectara a integrantes de pequeños productores y comunidades étnicas. Es decir, aquellos que, aun poseyendo la tierra, no cuentan con financiamiento y asistencia técnica para trabajarla y destinan su mano de obra a empleos transitorios o de bajos ingresos. Las políticas fueron transformándose a lo largo del tiempo, hasta la actualidad, en pos de generar una supuesta mejora de la población a partir del incremento de valor en las actividades que desarrollaran, ya sean agrarias o no. En este sentido, se trabajó en los programas de desarrollo rural, fundamentalmente, en el cuidado del medio ambiente, en la conservación de las especies naturales y en la consolidación de las organizaciones, insertándolos en el mercado económico.

Es decir, se crearon políticas a partir de la incorporación de dicha población rural (campesinos e indígenas) en estos mercados con el objetivo de "modernizar" sus labores. Paralelamente en estos proyectos se realizaron trabajos de asistencia técnica. Así, se convirtió a la población rural, en beneficiaria de tales mercados, dado que históricamente, se los ha considerado como "tradicionales y atrasadas que deben ser transformadas" (Lattuada 2014: 40). Es decir, se entiende a los integrantes indígenas y a los pequeños productores -cabe destacar que los pequeños productores se autoidentifican como no indígenas- como entidades puras y aisladas, descontextualizadas y ahistóricas, que pueden ser incorporados a la economía moderna a partir de inversiones en las economías tras el impulso de tales políticas de desarrollo.

\section{Politicas de desarrollo en pampa del indio}

A continuación, nos centraremos en la coyuntura que se presenta en Pampa del Indio -localidad ubicada al norte de la provincia del Chaco- y describiremos como es la implementación de tales políticas. Cabe aclarar que estas políticas no se elaboraron desde los organismos internacionales, teniendo en cuenta aquellos factores que llevaron a la pobreza de los sectores rurales. 
Es decir, no reconocen los contextos ni las situaciones con las que debe lidiar la población cotidianamente y las condiciones que generaron tal desigualdad. En un informe realizado por el Banco Interamericano de Desarrollo (Deruyttere 1997) sobre los pueblos indígenas y el desarrollo sostenible, se explican las razones por las cuales dicho organismo se encargó de trabajar con poblaciones en América Latina. Según el informe, la clave para explicar la pobreza en la Argentina era la pertenencia a un pueblo indígena: en este caso, el mapa de la pobreza en la Argentina, según el INDEC en el censo de 1991, revelaba que las zonas con más Necesidades Básicas Insatisfechas $(\mathrm{NBI})^{3}$ coincidían con aquellas donde habitaban comunidades indígenas. En dicho informe, realizado con el fin de ser presentado ante el Foro de las Américas del Banco Interamericano de Desarrollo en 1997, Deruyttere establece:

El Banco está emprendiendo iniciativas independientes formuladas específicamente para los pueblos indígenas, a fin de abordar los casos en que, debido al elevado grado de vulnerabilidad o la falta de oportunidades adecuadas de atención de las necesidades en las actividades regulares de la institución, los pueblos indígenas necesitan tener acceso a recursos específicamente destinados a atender sus demandas (Deruyttere 1997:14).

A pesar de lo dicho en este documento, las intervenciones de agencias de cooperación como el Banco Mundial, el Banco Interamericano de Desarrollo u otros organismos de orden local con financiamiento europeo, no lograron producir -en las comunidades donde trabajaban- cambios rotundos frente al escenario de conflictos producidos por la usurpación territorial o a los desajustes ambientales. Asimismo, a partir del análisis de este discurso podemos observar que en él no se contemplan los problemas estructurales de la implementación de dichas políticas (Ribeiro 1990, 1991). En el documento, se identifica a la población - en este caso los pueblos indígenas- como colectivos con problemáticas que deberían ser modificadas. Viola sostiene que " [...] el discurso del desarrollo despolitiza fenómenos como la pobreza, al definirla como un problema de los pobres y localizarla en un determinado sector de la sociedad, cuyas características intrínsecas servirían supuestamente para explicar la pobreza" (2000: 20). Es decir, tal como observamos en la cita de Deruyttere (1997), la vulnerabilidad y la pobreza son separadas del contexto que las produjeron, quitándoles su carácter político y transformándolas en un problema específico que posee la población indígena y en el cual los organismos de desarrollo deberán trabajar para reducir sus riesgos:

“Comprender mejor y tener en cuenta de manera más sistemática los impactos que producen en los pueblos indígenas el ajuste estructural y las reformas orientadas hacia el mercado, a fin de reducir al mínimo los riesgos y maximizar las oportunidades de desarrollo social y económico" (Deruyttere 1997:16) 
Recuperamos este informe del BID ya que da cuenta de la metodología que implementan las agencias de financiamiento internacional a la hora de trabajar con una realidad local que, pareciera ser ahistórica (Escobar 1995). Ahora bien, en este escenario donde la pobreza es estructuralmente significativa $-47 \%$ de la población de Pampa del Indio se encuentra por debajo de la línea de pobreza según los datos del INDEC (2010)-, los integrantes del pueblo qom se han organizado social y políticamente con el objetivo de demandar y reclamar por sus derechos y territorialidad. En este sentido, por un lado, el Estado (en sus diferentes niveles gubernamentales) acepta las inversiones externas a través de subsidios para la realización de tareas de forestación, extracción, turismo, etc. y, por otro lado, recibe tal financiamiento para "contener" a la población rural, que se organiza y reivindica por sus trayectorias y sus derechos territoriales y culturales. Es importante explicitar que gran parte de las organizaciones indígenas, como es el caso de Shiyaxauapi, 'Enauac, entre otras, se conformaron por miembros del pueblo qom de Pampa del Indio durante la década de 1980 y 1990, con el objetivo de encontrar soluciones a sus problemáticas cotidianas como así también el reclamo territorial y el acceso a los recursos. Desde este punto, las políticas de desarrollo rural se insertan como una "posible solución" a partir de la construcción de medidas que garanticen la participación de la población vulnerable de estas regiones en el mercado.

En la localidad aquí presentada, esta situación se vincula a la valorización del capital, en relación con la apropiación territorial y de los recursos que de ellos pueden extraerse. En ella conviven empresarios, fondos de inversiones internacionales, fundaciones, organismos gubernamentales y organizaciones etnopolíticas y campesinas. En la provincia del Chaco, específicamente en Pampa del Indio, los orígenes de los capitales que se orientaron a la explotación agroganadera son mayoritariamente nacionales, y convergen en proyectos comunales con bancos, actores comerciales y financieros (Albert 2000). En general, dichos programas de explotación tienen que ver con modelos de acumulación por desposesión (Harvey 2004). También se encuentra en estos modelos un proceso de internalización y transnacionalización, tal como definen Gorenstein y Ortiz (2016), donde los productores no solo poseen grandes extensiones de tierra, sino que también son parte de la cadena del agro, junto con otros empresarios de la misma envergadura. En este sentido, se dedican a la producción, el procesamiento y la comercialización de productos agropecuarios.

En la localidad aquí analizada trabajan grupos empresariales de gran renombre como Los Globo, Unitec y el equipo de la Familia Zaragoza ${ }^{4}$ Por un lado, se encuentran radicados la estancia La Leonor y Don Panos, 
los cuales pertenecen a la empresa UNITEC, presidida por Alfredo Karadagian. Además de las hectáreas pertenecientes a esta compañía, se encuentran la empresa Molinos Ríos de la Plata perteneciente a la familia Zaragoza. Todos ellos, sumada la empresa Grobo ${ }^{5}$ y Cargill Renovar, forman parte de la Cámara Argentina de Biocombustible (CARBIO), la cual se presenta como una de las mayores productoras de Biodiesel -gasoil realizado a base de Sojala cual exporta más del $80 \%$ a países como Estados Unidos y al continente europeo ([CARBIO], S/F). Según un informe del Ministerio de Economía en el año 2011, estos empresarios sumaban el 95\% de la producción de biodiesel ([Informe Ministerio Economía]. S/F). Además de las plantas para dicha producción, estas compañías son miembros en la asociación Round Table Responsible Soy (RTRS), que promueve la producción, procesamiento y comercialización de soja a nivel mundial, a partir de la entrega de certificados que garantizan la aplicación de procesos ambientales adecuados. Es decir, los mismos empresarios que poseen hectáreas en la localidad para la extracción de soja, forman parte de las corporaciones que certifican los productos, los procesan y exportan. Podemos entender en esta vinculación como aquello que Gorenstein y Ortiz (2016) llamaron internacionalización y transnacionalización a partir de la integración de grupos empresariales y actividades vinculadas.

\section{Agencias y políticas con financiamiento internacional y proyecto empresarial}

A partir del año 2003, en la república de la Argentina la situación económica había cambiado favorablemente respecto a décadas anteriores. En este contexto comienzan a efectivizarse nuevos préstamos por parte de aquellos organismos que, hasta ese momento, se habían retirado del escenario político económico argentino debido al endeudamiento existente (Tatuada 2014; Manzanal 2000).

Es nuestro interés presentar en este artículo el proyecto "Infraestructura Vial para el Desarrollo del Norte Grande" (de aquí en adelante DNG) implementado en la localidad de Pampa del Indio. El proyecto DNG se ejecutó a partir de la intervención del Ministerio Nacional de Planificación Federal de Inversiones ${ }^{6}$, el cual recibía fondos de organismos como el Banco Internacional de Reconstrucción y Fomento (BIRF), mejor conocido como el Banco Mundial.

El proyecto DNG a través del convenio 7991.AR se realizó en el año 2011 y constaba de un préstamo de 400 millones de dólares estadounidenses para la realización de obras de mejoramiento, ampliación y rehabilitación de 
tramos prioritarios de la red vial provincial, en la región del norte argentino. Entre los objetivos específicos que se presentan en el pliego del proyecto, encontramos: 1) Mejorar condiciones de accesibilidad y conectividad de los circuitos de actividad económica y productiva con mercados locales y externos; 2) Reducir los costos operacionales vehiculares y tiempos de viaje; 3) Aumentar la competitividad de las industrias de la región, entre otros objetivos ([Unidad de coordinación], S.F). En el informe elaborado por el grupo del Banco Mundial (BM) denominado "CAMINOS HACIA EL AUMENTO DE LA CAPACIDAD DE ACCIÓN. Iniciativa de la Ruta de la Cultura Qom, Provincia del Chaco - Argentina.

En este sentido, el Estado Nacional a través del Ministerio de Planificación, decidió realizar mejoras edilicias en la Ruta Provincial N. ${ }^{o} 3$ (RP3) [Ver imagen 2], que contiene el tramo Pampa del Indio- Villa Rio Bermejito, con los fondos asignados por el BM.

Imagen 2: En este mapa se puede observar un ovalo pequeño que da cuenta de la ubicación de la RP3 y un ovalo que lo contiene y que referencia el área de influencia del proyecto que considera a la región delimitada a lo largo de la RP4 y RP95.

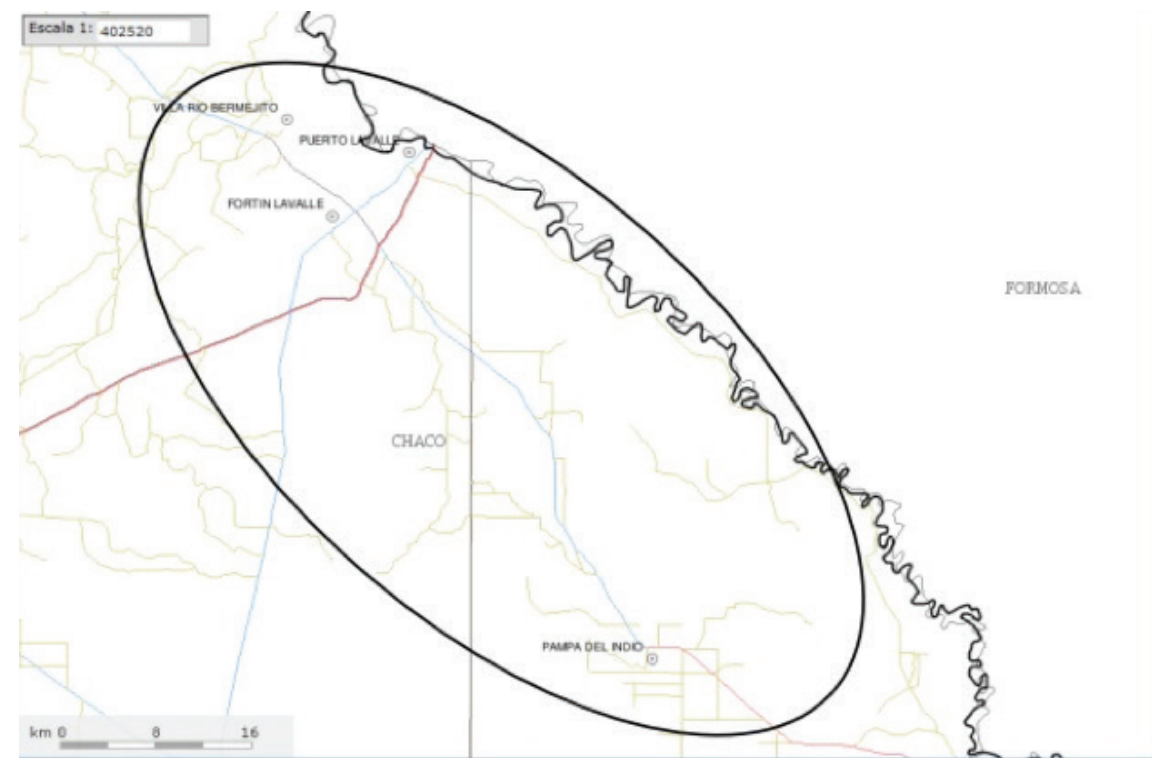

Mapa disponible en: Informe de Impacto medioambiental, S.F.

Para la pavimentación de este tramo, la suma monetaria destinada fue de 240 millones de pesos que se ejecutaron tras una evaluación social, en la cual miembros del Ministerio de Planificación relevaron la zona a intervenir y a las comunidades que se verían afectadas. El BM, para adjudicar este tipo 
de préstamos, posee una normativa donde especifica que en el momento que se realice una obra que atraviese una zona habitada por comunidades, se debe trabajar con ellas. Dado que existen poblaciones indígenas que viven a los márgenes de la RP3, se implementó el Plan Pueblos Indígenas (PPI). El objetivo de este plan es el de asegurar que las obras de los programas de infraestructura vial incluyan medidas para evitar efectos adversos sobre las comunidades. Según los documentos del BM, en el caso de que esto sea inevitable, buscarían reducir tales consecuencias indeseadas y otorgarles, a dichas poblaciones, beneficios sociales y económicos. A partir de esto, se confeccionó un circuito de identificación de comunidades con fines turísticos para que los usuarios, que transitan la RP3, conozcan las áreas donde habitan los pueblos originarios y los atractivos que ellas brindan. Catalina, ex integrante del equipo que coordinó la implementación del proyecto DNG, nos explicó en una entrevista que la salvaguarda que realiza el BM es muchas veces ejecutada en pos de proteger los intereses vinculados a las trasferencias de capitales y arreglos entre los entes gubernamentales:

"La asistencia de esta unidad, viene de los condicionamientos de los bancos para hacer los préstamos, no deriva de la intención de proteger el ambiente o los pueblos indígenas del área rural, más allá de lo que hace cualquier empresa de evitar que los rentistas 'molesten'. Esto era más riguroso porque el Banco Mundial tiene salvaguardas muy fuertes y está por encima de temas sociales e indígenas" (Catalina, Coordinadora del proyecto DNG, marzo 2016).

En este caso y en función de esta salvaguarda que exige trabajar con las comunidades que se vieran afectadas por las obras, el BM contrató a la Fundación Noreste Argentino (Fundación NEA), que trabaja en la región desde la década de 1990. La fundación NEA había sido contratada para el PPI con el fin de garantizar una integración de los pueblos étnicos a partir de la realización de la mejora vial. La Fundación de origen italiana está financiada por la Cooperación Italiana Nord Sud desde el año 1988 y por la Agencia de Naciones Unidas de la Comisión Europea, entre otras asociaciones y organizaciones que sustentan los trabajos. Esta Fundación es una institución de la sociedad civil sin fines de lucro que trabaja en la provincia de Formosa, Chaco y Salta y fue creada con el objetivo de promover el desarrollo de los pueblos del Gran Chaco, según sus documentos fundacionales. Entre estos se puede leer:

“La Fundación pretende apoyar el desarrollo de proyectos productivos que refuercen las actividades tradicionales propias de la población aborígenes y criollas (artesanías, apicultura, forestación, ganadería) enmarcándolas en la lógica del mercado, para que puedan llegar a generar ingresos continuos y mejorar las condiciones de vida de los productores en todos sus aspectos" ([Descripción Avina], S.F) 
POLÍTICAS DE DESARROLLO Y ORDENAMIENTO TERRITORIAL:

UN ANÁLISIS ACERCA DE LA VINCULACION DE AGENCIAS INTERNACIONALES

ORGANISMOS GUBERNAMENTALES Y NO GUBERNAMENTALES Y POBLACIÓN INDÍGENA

La Fundación Noreste Argentino no es la única asociación que trabaja en el territorio con las organizaciones étnicas, dado que uno de los objetivos de dicha Fundación es vincular a las comunidades con otros organismos y asociaciones para maximizar sus posibilidades. En este sentido, efectúa trabajos corporativos junto con la Asociación Civil para la Integración y el Desarrollo (ACID). ACID es una empresa social de cooperación fundada en el año 1990, que promueve el desarrollo social y económico en áreas rurales y urbanas. Junto con la Fundación NEA y la Fundación Avina7, llevan adelante el proyecto de "El futuro Está en el Monte" (EFEM) implementado en la Ruta de la Cultura Qom (RCQ).

El proyecto RCQ se basó en generar actividades comerciales entre las organizaciones indígenas de la región, en las cuales no solo pudieran explotar sus capacidades artísticas sino también su cultura. En este sentido, la RCQ se estableció como un proyecto de turismo étnico. Entre las estrategias del mercado, las ONG han desarrollado proyectos turísticos, tal como incentiva el PPI, con el afán de establecer ámbitos y actores susceptibles de ser atractivos en tal rubro: las comunidades indígenas en sus lugares de pertenencia. Es decir, se buscó explotar turísticamente, la imagen generada históricamente acerca de esta población como seres 'autóctonos', 'naturales' y 'originales'. De este modo se consolidó la imagen de un "otro indígena" que responde a una caracterización atractiva para el turismo occidental.

El turismo, en los últimos años, se ha convertido en uno de los pilares más importantes para los organismos de financiamiento y es una actividad masiva que ha demostrado ser económicamente rentable. Además, tiene la función de transformar el ocio en un producto económico, que genera que las condiciones materiales de existencia sean algo atractivo para los visitantes. Así, estos otros son transformados a partir de un proceso de homogeneización (Dachary, 2015).

Entre las actividades turísticas que ofrece la Fundación NEA, está la de recorrer la RCQ, la cual brinda diferentes atractivos 'autóctonos'. En estos ámbitos turísticos, los mapas implantados en la ruta [Ver Imagen 3] y elaborados por las mismas comunidades, dan cuenta a los turistas de los lugares sagrados y de los espacios transcendentales y distinguidos para el pueblo qom. De este modo, quienes la recorran podrán 'visitar los pueblos, caminar por territorios sacros, obtener artesanías nativas, realizar la molienda de algarroba junto con las mujeres cocineras, extraer palma para confeccionar tejidos, probar platos típicos o hacer avistaje de aves y animales nativos en los montes'. 
Imagen 3. Mapa realizado por las organizaciones étnicas de la localidad y reutilizado por el PPI financiado por el Banco Mundial, a partir de la implantación del proyecto RCQ.

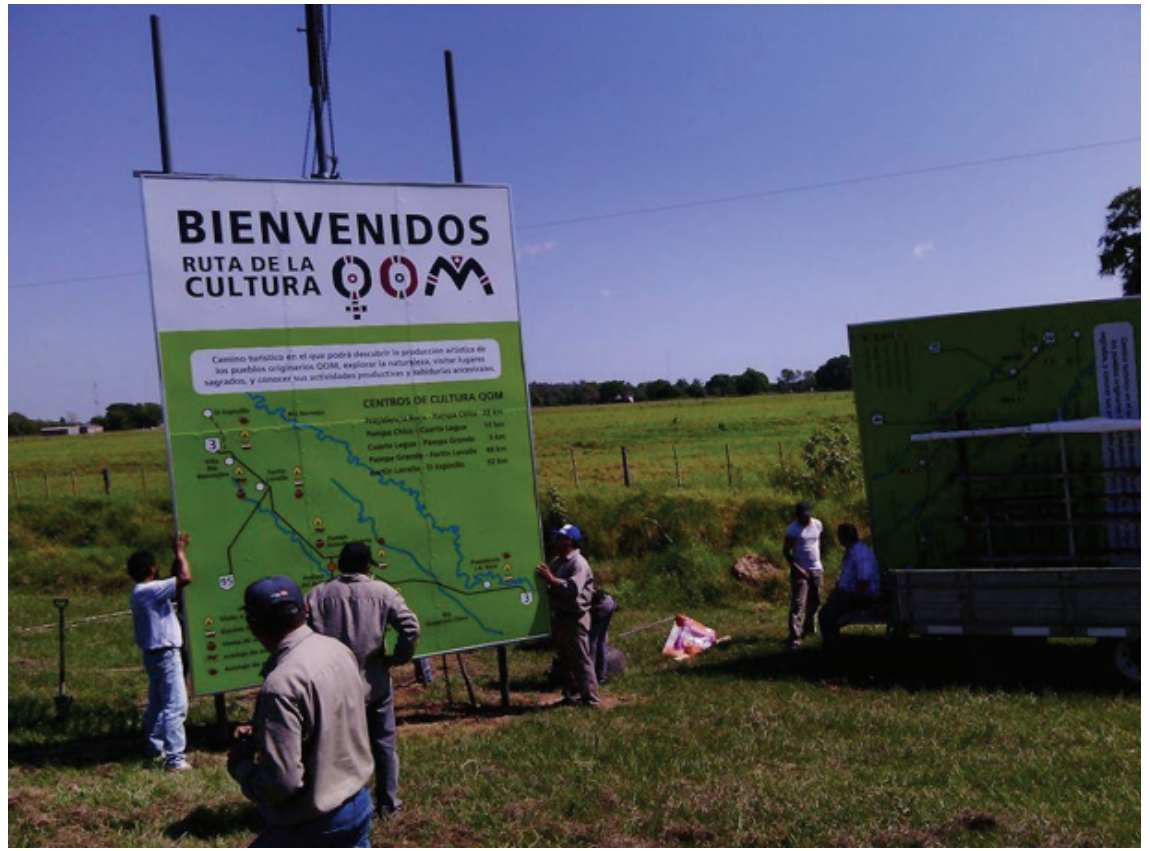

Fotografía tomada en el trabajo de campo de agosto 2017.

Cabe destacar que las fundaciones que impulsan estas actividades se encuentran vinculadas en una "red de redes", generada por sus presidentes o socios fundadores. Entre estas redes podemos mencionar que el fundador de ACDI -organización que impulsa y aglutina a las otras ONG en este proyecto de EFEM y desde el cual se diseña la RCQ- es el empresario sojero Jorge Varsovia. Asimismo, Varsovia, es socio fundador de la Fundación Avina, también involucrada en este proyecto. Para la realización y comercialización de artesanías la fundación NEA recurrió a la Fundación Artistas Nativos que trabaja en vinculación con dicha entidad.

“Flavia, nos decía de crear la Ruta de la Cultura Qom. Ahí empezamos desde la parte institucional a presionar cada uno desde una parte. Vialidad con los carteles de los mapas de los indígenas, el banco apoyaba con dinero, y Flavia quería crear una red de comercialización y un tejido social. Ella quería hacer turismo a partir de las artesanías con las comunidades, así empezamos" (Catalina, técnica del programa Norte Argentino, marzo 2016).

Artistas Nativos, trabaja en red junto con las otras asociaciones civiles y forma parte del proyecto turístico de la RCQ. Además, dicha Fundación 
está dirigida por una familiar cercana del presidente de ACID: Catalina, su exmujer. Como vemos, ACDI y Artistas Nativos están asociadas no solo por ingresos fiscales sino también por lazos de parentesco. Cabe destacar que gran parte de lo comprado por Artistas Nativos a las organizaciones indígenas, se comercializa en un showroom en unos de los barrios más costosos de la Ciudad Autónoma Buenos Aires: Palermo. Los productos también se venden en mercados y ferias de New York tales como Wanted Design a montos muy superiores a los que se pagaron a sus productores indígenas, según nos explicaron nuestros entrevistados. Tal como nos comentaba la presidenta y fundadora de esta organización, María Sol, el trabajo de la asociación se explica de la siguiente manera:

“Nosotros trabajamos en distintos territorios, y nuestra propuesta es tratar de ser una distribuidora de productos de base cultural. Trabajamos en una red de redes: para citarte un caso, trabajamos en el Chaco junto con la Fundación Nea, ACID, así como con el gobierno provincial, nacional, el Banco Mundial o el BID. Ellos han tenido mucha sensibilidad para escuchar cuales eran las necesidades y los ayudan. Por ejemplo, el INTI mismo, desarrollaron una máquina para el tenido de las fibras, otra para separarla y una desfibradora de chaguar, que nos sirven para la producción. Así también estuvo Samsung que hace un centro para que los artesanos se contacten con el mundo" (María Sol, presidenta de Artistas Nativos, noviembre 2016, la cursiva es nuestra).

Vemos en esta entrevista que la presidenta de la Asociación relata que su trabajo se genera a partir de la participación en una "red de redes" (Ver imagen 4) junto con otras ONG y organismos gubernamentales, que les permiten llevar a cabo su proyecto de comercialización. En este sentido, ella misma nos narraba como fue el proceso de trabajo junto con El Instituto Nacional de Tecnología Industrial (INTI), órgano que pertenece al Gobierno Nacional. A partir de la incorporación de la maquinaria entregada por este organismo a la Fundación, las comunidades empezaron a producir mayores cantidades de artesanías en menor tiempo y, de este modo, generó un aumento en las ganancias de la Fundación. Además, el INTI puso en funcionamiento un centro de servicios textiles de pueblos originarios en Formosa, a partir del cual se puede teñir la fibra con diferentes colorantes. Es decir, vemos como la fundación NEA y Artistas Nativos, se encuentran en una constante vinculación con organismos estatales que ofrecen su cooperación a partir del otorgamiento de recursos, maquinarias y técnicos que facilitan los procesos de producción de los proyectos implementados. 
Imagen 4. Cuadro de elaboración propia que grafica la red de redes que se distribuye en el territorio donde se detalla la relación que existe entre las fundaciones, los empresarios y sus firmas vinculadas a la cadena de producción en la que se encuentran. Cada uno de estos recuadros y sus vinculaciones se encuentra explicada en el texto aquí analizado y fue elaborado con la finalidad de generar un mapa conceptual que ayude al lector.

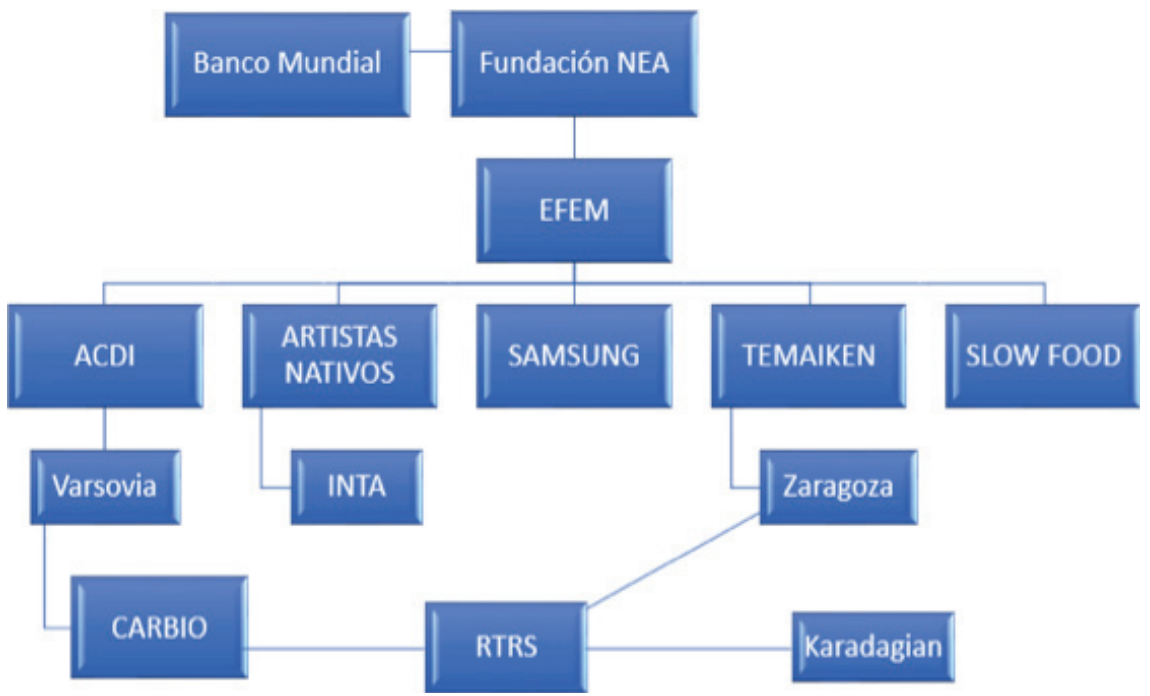

También en la entrevista, María Sol mencionó la participación que realizó la empresa Samsung Electronic Argentina. En Pampa del Indio, dicha empresa, a partir de la iniciativa "el Futuro está en el Monte", implementó el programa "Samsung Namún Village". En la página web de la empresa patrocinan su objetivo:

“Revertir las causas de la pobreza y establecer las bases para la independencia económica proporcionando la infraestructura integral necesaria para satisfacer las necesidades básicas en zonas desfavorecidas y de bajos recursos. El programa no solo mejora la infraestructura local mediante el trabajo en asociación con los gobiernos locales, sino que también alienta a nuestros empleados a involucrarse de manera activa en el desarrollo de las comunidades individuales" ([Empresa Samsung], S.F).

En este sentido, a partir de la donación de tablets y una computadora de escritorio a la organización indígena Shiyaxauapi, buscaron favorecer la integración y la comunicación de la organización con otros actores. |A partir de esta iniciativa, el centro comunitario de la agrupación étnica debía cambiar su nombre al de "Samsung Namum Village". Al respecto, Cintia, técnica de la Fundación NEA y responsable del trabajo en esta localidad, nos contaba lo siguiente acerca del proyecto: 
“Entre los proyectos está Samsung porque aparte lo que pretende con el Nanum es que sea como un ojo de la comunidad. En ningún lado dice que es como un ojo, pero eso se espera. Nanum es un proyecto con Samsung y desde la Fundación tienen como objetivo generar una red de productores, 'El futuro está en el monte' se llama en la Fundación. Negocios en red, lograr desarrollo desde el monte nativo y desde la revalorización de los saberes tradicionales que tienen los productores (...) el objetivo de estos centros es acercar innovación tecnológica a las comunidades aisladas del Gran Chaco" (Cintia, Fundación NEA, marzo 2016, la cursiva es nuestra).

Entre las otras actividades que ofrece la Ruta de la Cultura Qom está la de avistaje de aves que es impulsada por la fundación Vida Silvestre que comenzó a trabajar en Pampa del Indio junto con la Fundación Temaikén ${ }^{8}$. Esta última fundación, trabaja en pos de "proteger la naturaleza, educando, investigando y conservando especies, ecosistemas y priorizando los autóctonos" según establecen en un folleto que nos entregaron en un encuentro que concretamos en el Partido de Escobar, en el zoo que posee la Asociación. Cabe destacar que dentro del proyecto DNG financiado por el BM, uno de los requisitos era el de contar con equipos que sean responsables ambientales en las localidades donde se realicen las obras. Cuando les consultamos a los empleados de Temaikén acerca de estas cuestiones vinculadas a la privatización del monte y la consecuente extinción de su fauna y flora, Martín nos comentaba lo siguiente:

“El proyecto lo que hace es investigación, para hacer algo tenemos que saber... en paralelo nos traen un proyecto de investigación que es conservar el tapir en esta zona y a simple vista parece que el estado de conservación del monte está en buen estado, para si esto se mantiene así en un futuro hacer liberaciones (...) además, no surge el tema de las privatizaciones o fumigaciones, no como denuncia ni intervención política, quizá lo mencionan, pero no lo denuncian" (Martín, Temaikén, octubre 2017).

Ahora bien, los técnicos con los que nos entrevistamos en la Fundación plantean que la falta de conservación del monte es responsabilidad de las comunidades, y en este sentido realizan las capacitaciones y formaciones con los integrantes de esta para otorgarles a las poblaciones locales la necesidad de preservar las especies. De esta forma niegan la realidad territorial que las comunidades atraviesan. Además, esto invisibiliza no solo la situación vinculada a la usurpación territorial, sino que hace a un lado la realidad cultural que les es arrebatada a las comunidades, como producto del expansionismo de empresas agrícola ganaderas. En este sentido, la instalación de dichas industrias genera la apropiación territorial, la privatización de los montes, 
y por lo tanto la prohibición de actividades como la marisca y recolección para alimentarse o elaborar artesanías.

Nos interesa remarcar una cuestión antes de continuar y es que la Fundación Temaikén, está constituida y financiada por la familia Zaragoza, una de las familias más acaudaladas del país y que tiene negocios en la producción de biodiesel junto con Alfredo Karadagian y Jorge Varsovia en CARBIO, como mencionamos anteriormente, pero también forman parte, como miembros activos, de RTRS. Varsovia, fue elegido en el año 2003 presidente de esta asociación internacional, que garantiza el procesamiento y comercialización de la soja a nivel mundial ([RTRS elige nuevo Presidente] 2013). Además de estas relaciones que entablan en CARBIO y RTRS, los empresarios, la Fundación Temaikén tiene como proyecto en su parque zoológico ubicado en Escobar, instalar un sector donde la Fundación NEA y Artistas Nativos puedan comercializar las artesanías de la comunidad. Además, la fundación NEA tiene vinculaciones laborales con Temaikén a partir del proyecto El Futuro Está en el Monte, donde se proponen la producción de algarroba, para la exportación de los productos, junto con la Asociación de Artistas Nativos.

No nos resulta casual, entonces, que en este escenario se implemente el proyecto del BM para pavimentar una de las rutas que conecta el "anillo productivo" de la provincia. Ahora bien, dichos empresarios, como analizamos anteriormente, se instalaron en la zona, en gran medida, a partir de usurpaciones territoriales y de la compra de tierras a bajo costo o a partir de vinculaciones con organismos públicos.

Es importante en este sentido, dar cuenta que, a la lógica de acumulación y explotación territorial, se suman otros componentes que deben analizarse como parte del mismo proceso. En primer lugar, la intervención y regulación que tienen los diferentes organismos estatales. Un segundo componente vinculado a la ocupación que ejercen los empresarios sobre tierras productivas y la consecuente expulsión de población local. Por último, dicha población local, muchas veces conformada por integrantes de los pueblos indígenas y por pequeños productores, se encuentran sin herramientas y acompañamiento legal y jurídico para resolver estas situaciones.

En el primer caso, el proceso de apropiación de tierras en la provincia del Chaco se dio en connivencia con el gobierno provincial a partir del accionar que tiene el instituto de colonización, tal como vimos al comienzo. Es importante resaltar que, durante las últimas décadas, las agrupaciones indígenas y de pequeños productores se han familiarizado con el accionar y funcionamiento del sistema político y económico nacional e internacional. En este sentido, esta familiarización repercute en el liderazgo y organización, 
ya que su participación en tales proyectos y su alianza con estos espacios y organismos les permiten obtener mayor visibilidad y efectividad respecto a sus demandas (Viola 2000).

\section{Reflexiones finales}

Tal como vimos, desde la década de 1980, las medidas económicas ejecutadas en la región han generado una desestructuración de los modos de producción locales -tras la expansión de la frontera agroganadera-, la cual produjo la expulsión de gran parte de la población indígena de sus territorios. Aquel sector de la población étnica que, a pesar de ello, logró mantenerse en sus lugares de origen, fue incorporado -por el empresariado nacional e internacional- como mano de obra barata a la dinámica capitalista. En este sentido, hemos trabajado sobre las diversas transformaciones socioeconómicas, las inversiones privadas destinadas a la explotación de los recursos naturales y a la implementación de políticas públicas, como el PPI implementado por el BM junto a una red de fundaciones, afectaron diferencialmente a los integrantes del pueblo qom de la localidad. Estos cambios produjeron resignificaciones en el movimiento indígena que contribuyó a visibilizar e intensificar sus acciones, como también a generar nuevas vinculaciones y estrategias en la dinámica de relacionamiento con otros espacios de la sociedad civil.

Entre las políticas implementadas por los entes provinciales analizamos, entre otras, las parcelaciones que se realizaran a través de los alambrados, que, de algún modo, garantizan el avance de la frontera agrícola-ganadera. Asimismo, buscamos describir las dinámicas productivas impulsadas por la "red de redes" de empresarios vinculados en Pampa de Indio. Analizamos el modo en que los organismos gubernamentales -destinados a trabajar con población originaria- formularon y ejecutaron políticas, en estos escenarios desfavorables para la población, que crearon nuevos modos de habitar y producir el territorio. Por otro lado, describimos cómo dichos entes, al incrementar estas acciones (como las privatizaciones, por ejemplo) posibilitaron acciones alternativas, de resistencias y negociación entre los integrantes de los pueblos. Es decir, describimos aquellas políticas que buscaron invisibilizar y excluir a la población indígena a partir de la construcción de discursos exotizantes de la población étnica.

En tal sentido, creemos importante afirmar que, cuando los organismos gubernamentales y no gubernamentales elaboran discursos acerca de la 'inmutabilidad y pureza' indígena, se anulan sus trayectorias y las condi- 
ciones de existencia en las que se encuentran actualmente. Es decir, dichos entes, afirman que su labor estará destinada a trabajar en pos de revertir las condiciones de pobreza que sufren las comunidades, sin analizar las causas estructurales que las generaron. Sin embargo, pudimos observar las maneras en que la organización indígena, a partir del rol que se les asignó en tanto "colectivo exótico". Entre otros ejemplos analizamos aquí, la participación y el protagonismo que tuvo Shiyaxauapi a partir del proyecto de Norte Grande, el cual fomento un programa de turismo étnico. Vimos cómo, desde diversas áreas de ejecución (Temaikén, Samsung, Artistas Nativos, Fundación Gran Chaco, ACID, entre otras), se buscó explotar a las comunidades, a partir de su naturalización.

Nos parece importante afirmar que, allí donde fueron implementados estos proyectos con el objetivo de "empoderar económicamente" o "solucionar problemas de pobreza" -tal como afirman los técnicos de estas agencias-, se generaron acciones que obraron a favor de un sector empresarial que, muchas veces, es quien profundiza las condiciones de desigualdad. Son los empresarios -en los casos aquí trabajados- quienes, en connivencia con el Estado, intervienen en las comunidades para contener posibles conflictos. En este sentido, se crea un aparente consenso allí donde existe una disputa permanente, que invisibiliza las problemáticas históricas y las trayectorias de las comunidades que resisten a dicha diferenciación y pobreza estructural. Ahora bien, el empresariado -a partir de las usurpaciones territoriales y la compra/ventas de tierras fiscales- se ha instalado en la región, con un fin productivo y comercial. Este se vincula cotidianamente con los grupos indígenas a través de sus fundaciones y asociaciones civiles, con el objetivo de aminorar posibles conflictos, pero también para explotarlas como mano de obra barata para trabajar en sus campos y en los proyectos turísticos que tienen con tales, como la venta de artesanías por ejemplo. Es decir, dimos cuenta que los integrantes de esta "red de redes" utilizan aquellas herramientas creadas por las organizaciones indígenas con fines meramente comerciales y productivos, proporcionándoles, a través de estrategias de marketing, un sentido diferente con el que fueron creadas (por ejemplo, las artesanías).

A pesar de ello, nos parece importante señalar que, las organizaciones obtienen una fuente de ingresos a través de la venta de productos, y la presentación a nuevos proyectos o concursos, que si bien no modifica la pobreza en la cual están insertos, les brinda la posibilidad de obtener mayor visibilidad y reconocimiento para revertir dichas condiciones. Dichos grupos, consolidados a través del tiempo, se insertaron -desde el pleno conocimiento de las condiciones a las cuales estaban siendo expuestos- 
en las redes de explotación y producción, para resignificar 'desde adentro' su protagonismo. De este modo, es desacertado considerar que estas organizaciones, simplemente, son explotadas por dichos entes, dado que, en la dinámica diaria, ellas negocian y resignifican su participación en las redes de producción (Radovich y Balazote, 1995). Es decir, el proceso de consolidación de las organizaciones indígenas y su participación en proyectos de financiamiento internacional no se produjo de manera lineal y homogénea, sino que -al contrario- fue un proceso dinámico, cambiante y de constantes negociaciones. De este modo, acuerdo y conflicto, adaptación y resistencia, se marcan como palabras claves en este análisis.

Recebido em: 29 de agosto de 2019

Aprovado em: 03 de fevereiro de 2020

Malena Inés Castilla

Doctora por la Universidad de Buenos Aires (2019), orientación Antropología de la Facultad de Filosofía y Letras (FFyL-UBA). Es licenciada en Ciencias Antropológicas de la misma Facultad. Actualmente realiza su posdoctorado con financiamiento del Consejo Nacional de Investigaciones Científicas y Técnicas (CONICET).

https://orcid.org/0000-0002-1040-5568

Mail: malenacastilla@gmail.com 


\section{Notas}

1 "El Centro de Estudios e Investigación Social Nelson Mandela, se consolidó durante 1995 a partir de la participación de un grupo de reflexión y de discusión que se ocupaba de trabajar -como hacen actualmente- con los sectores más excluidos. En la actualidad, analizan las gestiones de gobiernos, las políticas públicas, los manejos de recursos, la evaluación de situaciones, entre otras. En este sentido, dicho centro se convirtió en una fuente de consulta para distintas organizaciones sociales, económicas, gremiales y políticas de la provincia del Chaco, como también fuente de colaboración para medios periodísticos locales, regionales, nacionales e internacionales" (Página web: La Matera de Guido 2008).

2 El total poblacional de la provincia del Chaco es de 1.055.259 habitantes. Ahora bien, de más de un millón de personas, 41.304 personas son integrantes de algún pueblo originario. Es decir, el 3,9\% de la población chaqueña se autoadscribe como descendiente o perteneciente a un grupo étnico. Los primeros pueblos mas significativos de la provincia en cuanto a su cantidad de habitantes son: el pueblo qom (toba) con un 74,5 \% de la población, el 11,2\% pertenecen al pueblo wichí y el 9,4\% al mocoví (INDEC, 2010).

3 Según la definición que da el INDEC en su página web, acerca del concepto de Necesidades Básicas Insatisfechas (NBI) permite delimitar a aquellos grupos que se encuentren bajo la línea de pobreza en términos estructurales, es decir, superando la idea de que la pobreza se produce por la falta de ingresos únicamente. En este sentido, la pobreza será entendida como privaciones materiales esenciales (INDEC, 2010).

4 La familia Zaragoza está compuesta por el empresario Francisco Zaragoza, su esposa "Magda", dueña de una importante cadena de heladerías -que elabora sus productos con vacas jersey- y sus siete hijos, uno de los cuales es quien se encarga de las empresas de la familia junto con su padre, quien figura en la revista Forbes como la cuarta persona más rica de Argentina.

5 El grupo Los Grobo, está presidida por el empresario Jorge Varsovia quien parte de un grupo económico que se dedica a la producción y explotación agroindustrial alimentaria. El grupo es una de las principales empresas en Argentina. Su presidente se encuentra primero en la producción de Trigo y segundo en la producción de soja, por el cual recibió el apodo de "el Rey de la Soja"

6 El Ministerio de Planificación Federal e Inversiones fue uno de los ministerios de Argentina durante diversas gestiones presidenciales. Hasta la asunción de Mauricio Macri como presidente de la Nación, el Ministerio estaba a cargo de la conducción de Julio De Vido, ministro que se mantuvo en su cargo durante los gobiernos de Néstor Kirchner (2003-2007) y Cristina Fernández de Kirchner (2007-2011 y 2011- 2015). 
7 “Fundación Avina es una fundación latinoamericana creada en 1994, enfocada en producir cambios a gran escala para el desarrollo sostenible de la región mediante la construcción de procesos de colaboración entre actores de distintos sectores para impactar de manera positiva en los Objetivos del Desarrollo Sostenible(...)Fundación Avina coordina la Plataforma de Innovación con Sentido, una alianza para acelerar el impacto social y promover una transformación a gran escala en favor del desarrollo sostenible en América Latina, a través de la combinación de la innovación social con la tecnológica, y la innovación en negocios" ([descripción Avina], S.F)

8 Temaikén comenzó su labor en la localidad tras la invitación realizada por COA MAÑIC (Club de observadores de aves) para trabajar en cuestiones vinculadas a la conservación ambiental y de las especies. El nombre de este bioparque deriva de los vocablos tehuelches "tem" (tierra) y "aikèn" (vida). 


\section{Bibliografia}

ALBERT, Bruce. 2000. "Associações indígenas e desenvolvimento sustentável na Amazônia brasileira." In RICARDO, Carlos Alberto (ed.): Povos Indígenas no Brasil, 1996-2000, pp. 197-207. São Paulo: Instituto Socioambiental.

BAINES, Stephen. 2001. As terras indígenas no Brasil e a "regularização" da implantação de grandes usinas hidrelétricas e projetos de mineração na Amazônia.

BAINES, Stephen. 2004. Antropologia do Desenvolvimento e a questão das sociedades indígenas. Revista Anthropológicas, 15(2).

DACHARY, Alfredo César. 2015. El turismo: un modelo de desarrollo. Revista Latinoamericana de Turismología, 1(1), 16-26.

DERUYTTERE, Anne. 1997. Pueblos indígenas y desarrollo sostenible: El papel del Banco Interamericano de Desarrollo. Inter-American Development Bank.

DIARIO NORTE. 2016 . Productores denuncian irregularidades en la adjudicación de tierras en la Reserva Criolla. 16 de Diciembre 2016. Recuperado de: http://www.diarionorte.com/article/146265/productores-denuncian-irregularidades-en-la-adjudicacion-de-tierras-en-la-reserva-criolla. (Fecha de ultima Consulta: 25 de julio de 2019)

DESCRIPCIÓN AVINA. s/f. Recuperado de: http://www.avina.net/avina/ fundacion/ (fecha de última consulta:16 de mayo de 2018).

ESCOBAR, Arturo. 1995. El desarrollo sostenible: diálogo de discursos. Ecología política, (9), 7-25.

ESCOBAR, Arturo. 1998. La invención del Tercer Mundo: construcción y deconstrucción del desarrollo. Editorial Norma.

GRILLO, Ralph. 1997. Discourses of Development: Anthropological perspectives. Nueva York, Estados Unidos: Berg Publishers.

GORENSTEIN, Silvia, \& ORTIZ, Ricardo. 2016. Empresas transnacionales en la agricultura y la producción de alimentos en América Latina y el Caribe. Documento de Trabajo, Fundación Foro Nueva Sociedad (NUSO), Buenos Aires, enero.

GUDYNAS, Eduardo. 2009. Diez tesis urgentes sobre el nuevo extractivismo. En Centro Andino de Acción Popular (CAAP) y Centro Latinoamericano de Ecología Social (CLAES) (Ed.). Extractivismo, política y sociedad (pp. 187-225). Recuperado de: http:// www.rosalux.org.ec/pdfs/extractivismo.pdf\#page $=187$.

HARVEY, David. 2004. El nuevo imperialismo (Vol. 26). Ediciones Akal.

HARVEY, David. 2007. Breve historia del neoliberalismo (No. 49). Ediciones Akal.

HOBART, M. (Ed.). 1993. An Anthropological Critique of Development. Londres, Argentina: Routledge

INDEC. 1991. Censo Nacional de Población y Vivienda 1991. Buenos Aires: Ministerio de Economía y Obras y Servicios Públicos de la Nación.

INDEC. 2001. Censo Nacional de Población y Vivienda 2001 Datos Provisionales. Buenos Aires: Ministerio de Economía y Obras y Servicios Públicos de la Nación.

INDEC. 2010. Censo Nacional de Población y Vivienda 2001 Datos Provisionales. Buenos Aires: Ministerio de Economía y Obras y Servicios Públicos de la Nación. 
POLÍTICAS DE DESARROLLO Y ORDENAMIENTO TERRITORIAL

UN ANÁLISIS ACERCA DE LA VINCULACION DE AGENCIAS INTERNACIONALES

ORGANISMOS GUBERNAMENTALES Y NO GUBERNAMENTALES Y POBLACIÓN INDÍGENA

INFORME MINISTERIO DE ECONOMÍA s/f. Recuperado de: https:// www.economia.gob.ar/peconomica/ docs/Complejo_Oleaginoso.pdf (fecha de última consulta:15 de mayo de 2018).

INFORME DE IMPACTO MEDIOAMBIENTAL s/f. Recuperado de: http:// www.ucpypfe.gov.ar/BirfPIVNGEIA.html (fecha de última consulta:15 de mayo de 2018).

LATTUADA, Mario. 2014. Políticas de desarrollo rural en la Argentina. Conceptos, contexto y transformaciones. Temas y debates, (27), 13-47.

MANZANAL, Mabel. 2000. Neoliberalismo y territorio en la Argentina de fin de siglo. Economía, sociedad y Territorio 2(7), 433-458.

MANZANAL, Mabel, ARZENO, Mariana, \& NARDI, Maria Andrea. 2010. Desarrollo, territorio y desigualdad en la globalización: Conflictos actuales en la agricultura familiar del nordeste de Misiones, Argentina. Mundo agrario, 12 (23).

MUÑOZ, Roberto, \& GALLO, Sergio. 2012. Conflictos por la tierra: El caso de la Unión Campesina del Chaco 2002-2011. In VII Jornadas de Sociología de la UNLP 5 al 7 de diciembre de 2012 La Plata, Argentina. Argentina en el escenario latinoamericano actual: Debates desde las ciencias sociales. Universidad Nacional de La Plata. Facultad de Humanidades y Ciencias de la Educación. Departamento de Sociología.

RADOVICH, Juan Carlos, \& BALAZOTE, Alejandro. 1995. Transiciones y fronteras agropecuarias en Norpatagonia. Producción doméstica y ca- pital. Estudios desde la Antropología Económica, 63-79.

RIBEIRO, Gustavo. 1990. "Da prefeitura ao Banco Mundial: para uma metodologia de ação política com relação aos grandes projetos." Série Antropologia, 97, Brasília: Departamento de Antropologia, UnB.

RIBEIRO, Gustavo. 1991. Empresas transnacionais: um grande projeto por dentro. São Paulo: Marco Zero/ ANPOCS.

RIBEIRO, Gustavo., y ESCOBAR, Arturo. 2006. Las antropologías del mundo: transformaciones de la disciplina a través de los sistemas de poder. Universitas humanística, volumen 61, pp. 15-50.

RTRS elige nuevo presidente. 2013. Recuperado de: http://www.losgrobosgr. com.ar/novedades/627—rtrs-eligenuevo-presidente.html (fecha de última consulta:11 de junio de 2018).

SCHMIDT, Mariana. 2014. Territorio (s), desarrollo (in) sustentable y naturaleza colonizada. Una propuesta de abrdaje conceptual. Pampa: Revista Interuniversitaria de Estudios Territoriales, (10), 101-130.

TRINCHERO, Hector Hugo. 2007. Aromas de lo exótico (retornos del objeto). Para una crítica del objeto antropológico y sus modos de reproducción. Buenos Aires, Argentina: Editorial SB.

VIOLA, Andreu. 2000. La crisis del desarrollismo y el surgimiento de la antropología del desarrollo. En A. Viola (Comp.), Antropología del desarrollo: Teorías y estudios etnográficos en América Latina (pp. 5-9). Barcelona: Paidós. 


\section{POLÍTICAS DE DESARROLLO Y ORDENAMIENTO TERRITORIAL: \\ UN ANÁLISIS ACERCA DE LA VINCULACION DE AGENCIAS INTERNACIONALES, ORGANISMOS GUBERNAMENTALES Y NO GUBERNAMENTALES Y POBLACIÓN INDÍGENA}

\footnotetext{
Resumen

En el presente artículo, tenemos el objetivo de analizar el modo en que el capital internacional, junto con la administración pública local, provincial y nacional, intervienen en los territoritos e implementan diferentes políticas públicas que afectan diferencialmente a la población indígena local. Para ello, nos centraremos en un primer momento en el análisis de las políticas de desarrollo ejecutadas en países del denominado "Tercer Mundo", para más adelante analizar las medidas implementadas en la provincia del Chaco particularmente. En este sentido, analizaremos de qué manera organismos gubernamentales de la provincia intervienen en el ordenamiento territorial favoreciendo, en muchos casos, a los empresarios del agro. Finalmente, describiremos un proyecto financiado por el Banco Mundial denominado "Desarrollo Norte Grande", el cual se implementó en la localidad de Pampa del Indio, ubicada al norte de la provincia. A partir del análisis de este proyecto, podremos responder a nuestro objetivo y describir las vinculaciones entre las agencias de crédito internacional, el Estado nacional argentino, los empresarios transnacionales y la población étnica qom de Pampa del Indio. Palabras clave: Políticas, Desarrollo, Gobierno, Organismos, Indígenas, Agronegocio.
}

DEVELOPMENT POLICIES AND TERRITORIAL PLANNING:

AN ANALYSIS OF THE LINKAGE
OF INTERNATIONAL AGENCIES,
GOVERNMENT AND NON-
GOVERNMENT ORGANIZATIONS
AND INDIGENOUS POPULATIONS

Abstract

In this article, we analyse how international capital, along with the local, provincial and national public administration, intervenes in indigenous territories and implement different public policies that differentially affect the local indigenous population. We initially analyse development policies implemented in countries of the so-called "Third World", and then turn to measures implemented in the Chaco Province. In this sense, we will investigate how the government agencies of the province intervene in territorial planning, favouring, in many cases, agricultural entrepreneurs. Finally, we describe a project financed by the World Bank called " Big North Development" which was implemented in the town of Pampa del Indio, located in the north of the province. We thus describe the links between international credit agencies, the Argentine national State, transnational entrepreneurs and the Qom ethnic population of Pampa del Indio.

Keywords: Politics, Development, Government, Organizations, Indigenous, Agribusiness 
POLÍTICAS DE DESENVOLVIMENTO

E PLANEJAMENTO TERRITORIAL:

UMA ANÁLISE SOBRE

A ARTICULAÇÃO DE AGÊNCIAS

INTERNACIONAIS, ORGANIZAÇÕES

GOVERNAMENTAIS E NÃO

GOVERNAMENTAIS E POPULAÇÃO

INDÍGENA

\section{Resumo}

Neste artigo, temos como objetivo analisar a forma como o capital internacional, junto à administração pública local, provincial e nacional, intervém nos territórios e implementa distintas políticas públicas que afetam diferentemente a população indígena local. Para isso, vamos nos concentrar inicialmente na análise de políticas de desenvolvimento implementadas em países do chamado "Terceiro Mundo", para posteriormente analisar as medidas implementadas na província do Chaco em particular. Nesse sentido, analisaremos de que maneira órgãos governamentais da província intervêm no planejamento territorial favorecendo, em muitos casos, os empresários agrícolas. Finalmente, descreveremos um projeto financiado pelo Banco Mundial denominado "North Big Development", que foi implementado na cidade de Pampa del Indio, localizada ao norte da província. A partir da análise deste projeto, poderemos responder ao nosso objetivo e descrever as ligações entre as agências internacionais de crédito, o Estado nacional argentino, os empresários transnacionais e a população qom étnica de Pampa del Indio. Palavras-chave: Política, Desenvolvimento, Governo, Organizações, Indígenas, Agronegócio. 